\title{
Influence of perturbations produced by Electromagnetic Interference (EMI) in arc fault detection
}

\author{
E. M. Calderon, P. Schweitzer, C. Bonnet, S. Weber \\ Lorraine University, CNRS, Institut Jean Lamour (IJL), Nancy F-54000, France
}

\begin{abstract}
Our work focuses on the development of a test procedure and the creation of a database of current signature in presence of electromagnetic interference (EMI). These signals can affect the reliability of detection algorithms producing false tripping; therefore increasing the number of false detections. An experimental test bench allows generating series arc faults for different load configurations (UL 1699 and IEC 62606 standards). EMI disturbances are produced by the EMTEST UCS500 M4 generator.

All tests are performed with conventional household appliances (halogen lamp, vacuum cleaner, drill or computer). We rely on the EN 61000-4-4, EN 61000-4-5 and EN 61000-4-11 standards to emulate the main EMI disturbances presented in a home electrical network (lightning-type overcurrent, voltage variations, motor starts, etc.). Spectral analysis of these signals shows that EMI and the series arc spectra are comparable and can disrupt the operation of conventional detection algorithms generating considerable false detections
\end{abstract}

\section{INTRODUCTION}

$\mathrm{T}$ he prevention of fires in domestic electrical installations particularly related to the appearance of an electric arc is provided by an AFCI [1]. In order to be as effective as possible, the detection rate in face to series arcs must be as high as possible while minimizing the rate of false detections. The tests to be carried out to develop an AFCI circuit breaker are described in the UL 1699 standard for USA [2] and the IEC 62606 standard for Europe [3]. Generally the detection in an AFCI is based on the analysis of the current or the line voltage of the circuit to be protected. Some authors [4] analyzed EMI/RFI (radiofrequency interference) generated by industrial systems and compared EMI intensities to those with series arc faults in the frequency band below $1 \mathrm{MHz}$. In this work, we focus our attention on situations that may lead to false detections and more precisely to electromagnetic disturbances in home electrical networks. The authors have observed that a growing number of household electronic products generate electromagnetic interference capable of perturbing the proper function of common arc-fault circuit interrupter devices.

The main EMI disturbances that can be generated as well as the associated domestic loads are presented in the second part. We also present in the third part, the tests defined by EN $61000(4-4 ; 4-5 ; 4-11)$ standards [5]-[7] focusing on fast transient waves (BURSTs), surge, voltage dips and variations. In the fourth part, we analyze EMI perturbations in time and frequency domain and compare them with arc faults. Given that most AFCI devices operate at frequencies below $500 \mathrm{kHz}$ and exhibit a narrow bandwidth, the frequency analysis we performed was restricted to that similar frequency range. We employed a current probe with a sampling frequency of 1 $\mathrm{MHz}$ for the collection of this data.

\section{EMI PERTURBATIONS AND EXPERIMENTAL SETUP}

In the context of this paper, a perturbation is defined as an undesirable electrical disturbance able to degrade or disrupt the normal operation of a household electrical device. The origin and nature of such perturbations is broad, but all of them tend to fall into two general categories: conducted interference and radiated interference. The conducted perturbations use conductive materials as propagation paths, such as power cables for example. The radiated perturbations are transmitted by an electromagnetic wave through dielectric materials.

Most household electrical appliances are considered source of EMI perturbations. Conducted perturbations are injected directly or indirectly into a conventional home electrical network [8]-[12]. The classification of EMI perturbations is done taking into account the power operation of appliances and can be classified in two types as was done in [13]:

EMI sources due to industrial and commercial equipments

- Air conditioning

- Computer and switching circuits

- Fluorescent lamps

EMI sources due to consumer devices

- Refrigerators / freezers

- Mixers

- Washing machines

- Vacuum cleaner

- Light dimmers

All these equipments produce also electrical and magnetic radiated emissions. TABLE I gives the electric and magnetic densities levels at $30 \mathrm{~cm}$ from 115 Volt home electrical appliances [14].

TABLE I.

ELECTRIC AND MAGNETIC DENSITIES.

\begin{tabular}{|l|l|l|}
\hline Appliances & $\mathrm{E}(\mathrm{V} / \mathrm{m})$ & $\mathrm{B}(\mathrm{mT})$ \\
\hline Boiler & 130 & $0.15-0.5$ \\
\hline Refrigerator & 60 & $2-20$ \\
\hline Hair dryer & 40 & $0.08-9$ \\
\hline Vacuum cleaner & 16 & $3.5-30$ \\
\hline
\end{tabular}

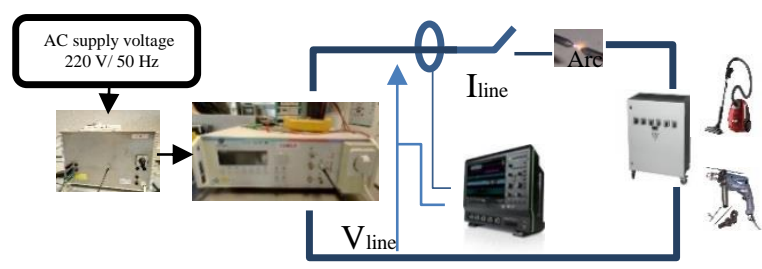

Figure 1. Experimental test bench.

In this paper, we focus only on conducted perturbations because arc fault detection algorithms in conventional AFCI 
deal mainly with the analysis of the current line. EMI perturbations are obtained using an experimental test bench shown in Fig. 1.

The test bench emulates a home electrical network into which EMI may be intentionally introduced via the EMTEST UCS500 M4 generator. The current and voltage of the line are measured at the output of the generator. The characteristics of the current and voltage probes are given in TABLE II. Data acquisition is carried out by a Lecroy oscilloscope wavepro $950(1 \mathrm{GHz})$ at $1 \mathrm{MHz}$ sample rate.

TABLE II.

PROBES CHARACTERISTICS.

\begin{tabular}{|l|l|}
\hline Voltage probe & Current probe \\
\hline \hline Lecroy PPe 20kV & Lecroy AP015 \\
$100 \mathrm{MHz}$ BW & DC 50MHz \\
Max. peak 20kV & Max. peak 50A \\
\hline
\end{tabular}

Series arc faults are inserted in the line in simple and masking configurations (Fig. 2) according to the UL 1699 and IEC 62606 standards. For arc fault generation through opening contacts, two copper electrodes of $6 \mathrm{~mm}$ in diameter are used for different tests.

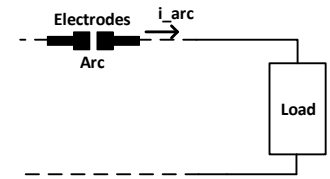

a) Simple.

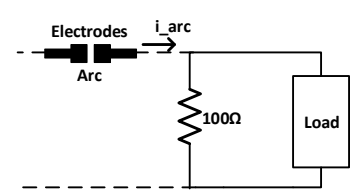

b) Masking.
Fig. 2. Load configurations.

\section{STANDARDIZED EMC TESTS}

EMC standards are used for product certification. These standards specify test methods and parameters to prove the immunity of an equipment or system in face to EMI perturbations.

We will generate EMI perturbations using the EN 61000-44, EN 61000-4-5 and EN 61000-4-11 standards which are detailed in the following subsections.

\section{A. EN 61000-4-4 standard-Fast transient immunity (BURST)}

This test couples BURSTs composed of a number of fast transient to the equipment power ports. The significant elements of this test are the high amplitude, the short rise time, the repetition frequency and the low transient energy.

The waveform used in this test standard is representative of disturbances that may be caused by switching transients (inductive load interruption, rebound of relay contacts, computer circuits, spark in air produced by unplugging, etc.). The characteristics of a BURST are:

- Rise time: $(5 \pm 1.5) \mathrm{ns}$,

- Pulse width: $(50 \pm 15) \mathrm{ns}$,

- Burst duration: $(15 \pm 3) \mathrm{ms}$ at $5 \mathrm{kHz}$,

- Burst period: $(300 \pm 60) \mathrm{ms}$,
- Peak voltage: 0.5 to $4 \mathrm{kV}$ (according to the level of severity).

Fig. 3 displays the train of pulses BURSTs and shows details of a BURST wave.

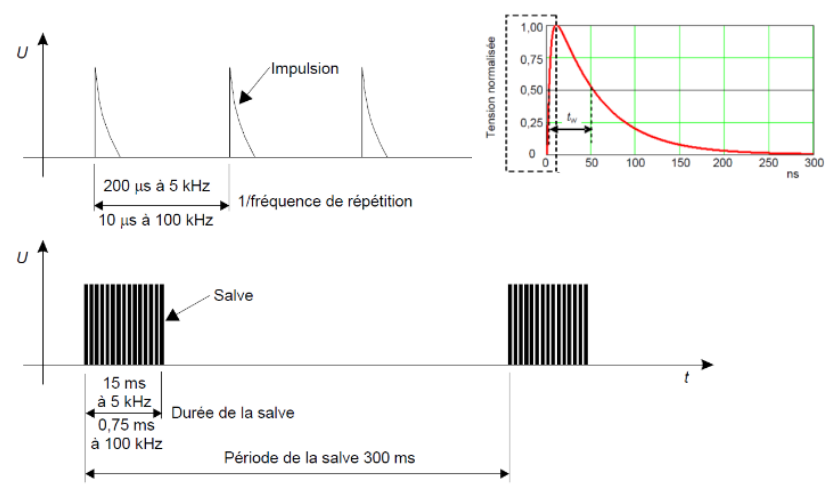

Fig. 3. BURSTs signal.

\section{B. EN 61000-4-5 standard-Surge immunity}

This test evaluates the effects of the transients associated with power system switching (such as capacitor bank switching), switching devices (e.g. transistors, thyristors, etc.), various system faults (short circuits...) as well as surge voltages produced by lighting stroke (direct or indirect stroke).

In TABLE III, parameters for open-circuit voltage and short-circuit current are specified.

TABLE III.

SURGE PARAMETERS

\begin{tabular}{|l|c|c|}
\hline & Front time (T1) & Duration (T2) \\
\hline $\begin{array}{l}\text { Open-circuit } \\
\text { voltage }\end{array}$ & $1.2 \mu \mathrm{s}$ & $50 \mu \mathrm{s}$ \\
\hline $\begin{array}{l}\text { Short-circuit } \\
\text { current }\end{array}$ & $8 \mu \mathrm{s}$ & $20 \mu \mathrm{s}$ \\
\hline
\end{tabular}

The surge is applied directly from the generator output terminals according the specifications in Table III. Fig 4 shows the voltage waveform of a surge at $1 \mathrm{kV}$.

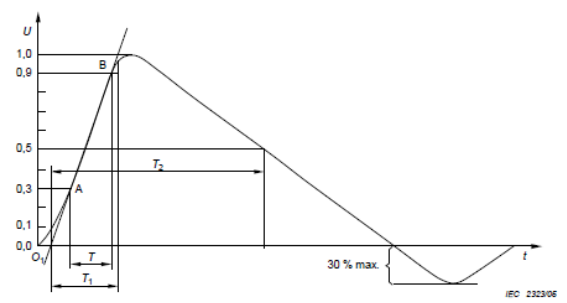

Fig. 4. Open circuit voltage representation.

\section{EN 61000-4-11 standard-voltage dips and voltage variations immunity}

This test evaluates the effects of short dips and breaks of equipment power supply caused by faults in the network, primarily short circuits or sudden large changes of load. These phenomena are characterized in term of the deviation from the rated voltage and duration. Voltage dips are simulated over a period of $500 \mathrm{~ms}$ for a voltage level of $70 \%$ of the rated voltage.

For voltage variations, the voltage decreases linearly to the reduced voltage and then goes up linearly to the nominal 
value. This is the typical power supply profile when a motor starts. The rise and fall times are in the order of $2 \mathrm{~s}$ and the reduced voltage is $70 \%$ of the rated voltage.

In Fig. 5 is ilustrated a typical theorical voltage dip.

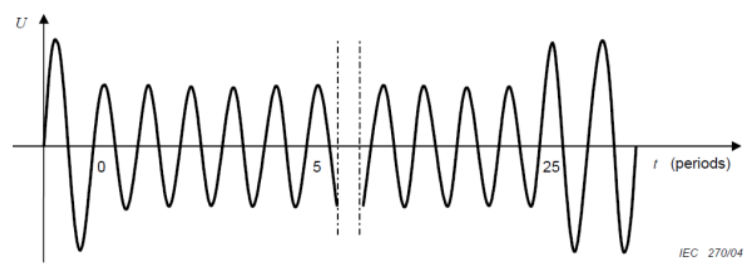

Fig. 5. Voltage dip.

\section{INFLUENCE OF EMI PERTURBATIONS}

The influence of EMI perturbations are presented in this section. In the experimental test bench, different household appliances are connected in simple and masking configurations according to TABLE IV. Waveforms in the time domain and spectral densities of recorded currents are obtained. The scope of our study is limited to a frequency band up $100 \mathrm{kHz}$ as is typical of AFCI current sensors.

TABLE IV.

LOAD USED IN THE EXPERIMENTAL TEST BENCH.

\begin{tabular}{|c|l|c|}
\hline Configuration & \multicolumn{1}{|c|}{ Appliance } & Power [W] \\
\hline Simple & Resistor $=100 \Omega$ & 1500 \\
& Vacuum cleaner & 1600 \\
& 1 PC & 600 \\
& Drill & 400 \\
& Halogen lamp & 500 \\
\hline Masking configuration & Vacuum cleaner // Resistor & \\
& Drill // Resistor & \\
\hline
\end{tabular}

\section{A. Temporal analysis}

The surge emulation (EN 61000-4-5 standard) is produced in the circuit by the EMI generator supplying a resistive load. A surge event is generally considered as a non-repetitive phenomenon in a short time interval. The surge voltage inserted in the experimental power line produces variations in the line current (Fig. 6).

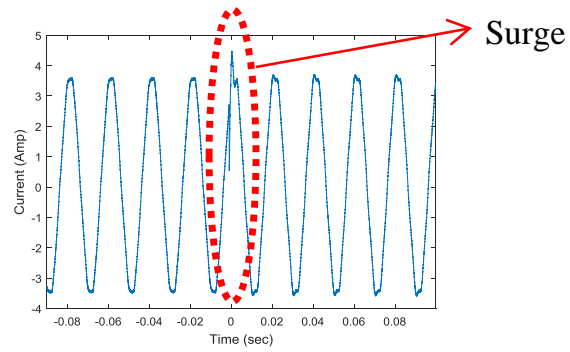

Fig. 6. Resistor current.

The disturbance causes a current variation (visible at $\mathrm{t}=0 \mathrm{~s}$ ) of rather short duration (a half cycle).

The tripping of an arc fault circuit breaker can only be done in presence of a fault maintained over several periods of the signal. Surge perturbation cannot be interpreted as an arc fault.

On the other side the BURST, voltage dip and voltage variations generated according EN 61000-4-4 and EN 610004-11 standards are inserted in our experimental power line using a vacuum cleaner, $1 \mathrm{PC}$, a drill and a halogen lamp as loads.

Fig. 7a shows the current line of a halogen lamp in stationary operation mode in simple load configuration. A BURSTs sequence with duration of $100 \mathrm{~ms}$ is capable to disturb the proper functioning of the appliance. In Fig. 7b, BURSTs are inserted periodically in the transient operation mode of a vacuum cleaner. A BURSTs sequence with a time duration fixed to $200 \mathrm{~ms}$ is firstly injected in section A (transient part), then in section B (transition between the transient and stationary part) and finally in section C (steady state). BURSTs produce significant signal distortion.

In Fig. 7c is illustrated the current line of a vacuum cleaner in transient operation in masking load configuration. The temporal evolution of the current is constituted by a transient part, a transition state and a steady state part. A BURSTs sequence of $200 \mathrm{~ms}$ is injected in this last part. The masking effect reduces the signal distortion.

Fig. 7d displays the current and voltage in stationary operation for a PC. A voltage dip generation of $500 \mathrm{~ms}$ produces current with slight incremental amplitude variations and transient behavior. A current spike is observed at $0.58 \mathrm{~s}$. On the other side, in Fig. 7e the current in stationary operation for a vacuum cleaner is shown. In this case, the current amplitudes drop abruptly by the injection of a voltage dip. In the graphic, amplitudes in sections $\mathrm{A}$ and $\mathrm{B}$ have average values of $10 \mathrm{~A}$ and $12 \mathrm{~A}$ respectively. The transient effect in the current induced by a voltage dip can significantly affect the AFCI performance.

In Figs. $7 \mathrm{f}$ and $7 \mathrm{~g}$ are displayed the transient current for a PC (simple load configuration) and a vacuum cleaner (masking load configuration). The $2 \mathrm{~s}$ voltage variation inserted in the circuit produces current amplitude variations. In Fig. 7f the current of the PC has incremental amplitudes (above 5A) and an opposite trend with respect to the voltage variation. However, in Fig. $7 \mathrm{~g}$ the current variations have the same trend as the voltage variation. Unlike voltage dip, the voltage variations are inserted smoothly; however, they can also generate important disturbances that are able to trigger false trips.

Fig. 8 shows a comparison between the BURST and a series arc fault inserted in the current line (for a PC). The distortions obtained in the time domain are comparable. In this context, EMI perturbations can really affect the good AFCI performance.

\section{B. Spectral analysis}

Spectral analysis is generally used in the literature to identify series arc faults by comparing the spectral density of measured current in normal and in fault operations. Fault detection methods based on the energy variation resulting from the spectral coefficients is performed in a variety of bands ranging from low frequencies up to $20 \mathrm{kHz}$ [15]-[19]. However, EMI perturbations can also produce spectral densities comparable to arcing faults in this frequency band and can lead to false detections of AFCI.

In Fig. 9 are displayed different spectral densities for currents obtained from tests using appliances presented in TABLE IV. 


\section{BURST}

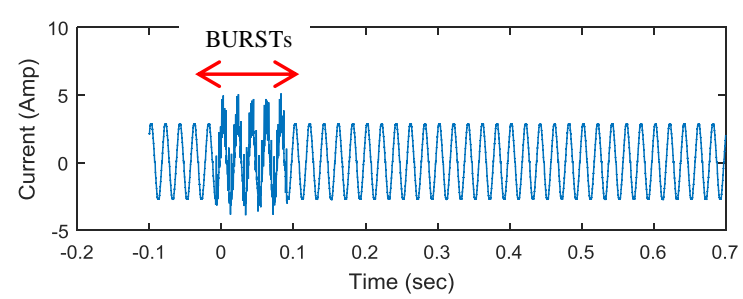

a) Halogen lamp

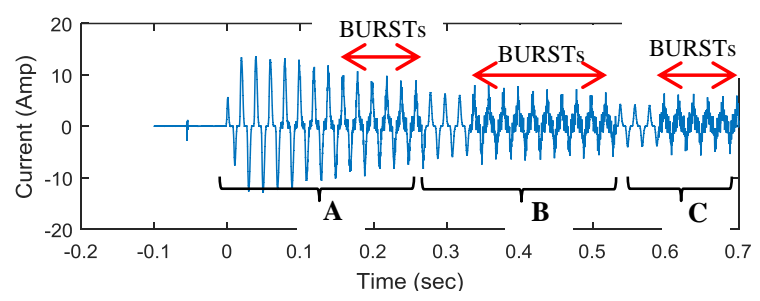

b) Drill.

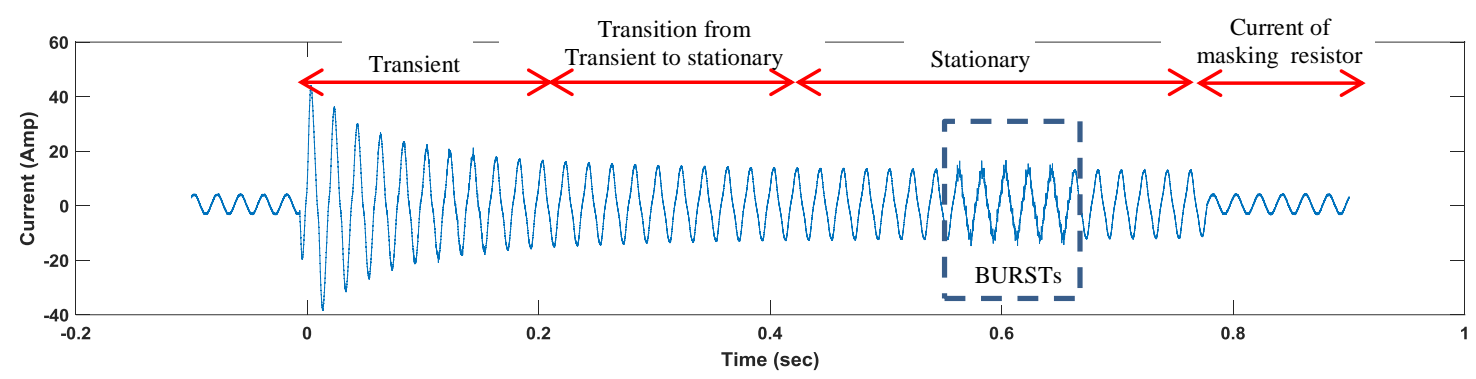

c) Vacuum cleaner - masking configuration.

\section{VOLTAGE DIP}
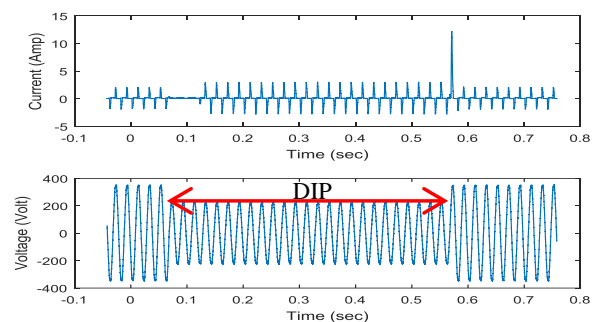

d) $1 \mathrm{PC}$.

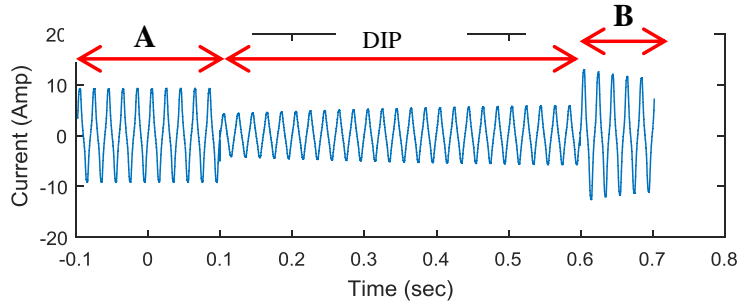

e) Vacuum cleaner.

\section{VOLTAGE VARIATION}

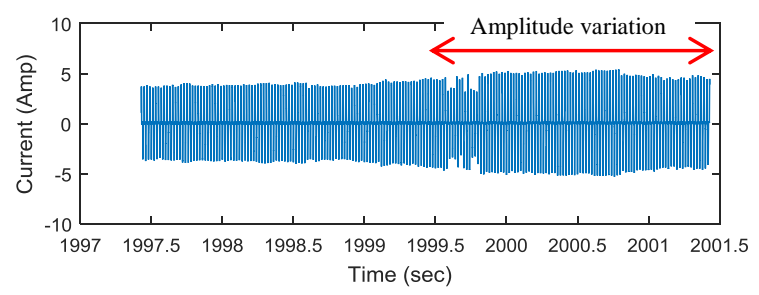

f) Current variation (1PC).

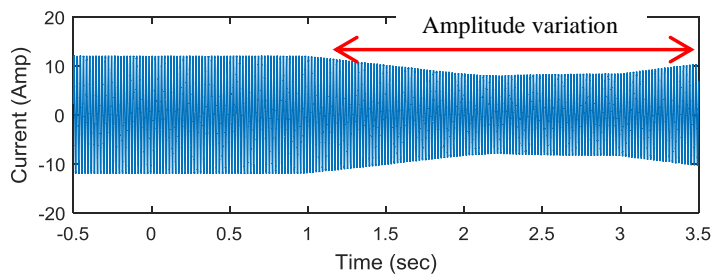

g) Current variation-masking configuration (Vacuum cleaner).

Figure 7. Current of the line considering differents EMI perturbations.

The spectral density for a PC in simple configuration shows an important influence of the BURSTs in comparison to voltage dip and voltage variations (Figs. 9a, 9d, 9g). In this regard, series arc faults can only be distinguished from BURST signal in a frequency band around $10 \mathrm{kHz}$.

The spectral density for a vacuum cleaner (Figs. $9 \mathrm{~b}$ and $9 \mathrm{~h}$ ) presents significant EMI perturbations which are difficult to identify from series arc faults. The BURSTS and voltage variations generated taking into account simple circuit configuration make difficult the series arc fault identification. The frequency band of $10 \mathrm{kHz}-100 \mathrm{kHz}$ is not representative to confirm the arc fault presence. In the case of voltage dip (Fig 9b), an arc fault can be identified using a frequency band of $20 \mathrm{kHz}-100 \mathrm{kHz}$. In the case of masking load configuration (Fig. 9i) tested under voltage variations, the masking effect produced by the $100 \Omega$ resistor, reduces the influence of EMI perturbations. In this case the arc fault identification is possible in a frequency band of $50 \mathrm{kHz}-100 \mathrm{kHz}$. 
The spectral density for a drill (Fig. 9f) shows EMI perturbations originated by voltage dip in simple circuit configuration. In this case a series arc fault is identified in the frequency band of $10 \mathrm{kHz}-100 \mathrm{kHz}$.
Finally the most difficult case is presented in Fig. 9c (simple configuration) for a halogen lamp in which is not possible to identify a series arc fault from EMI perturbations produced by the BURSTs.

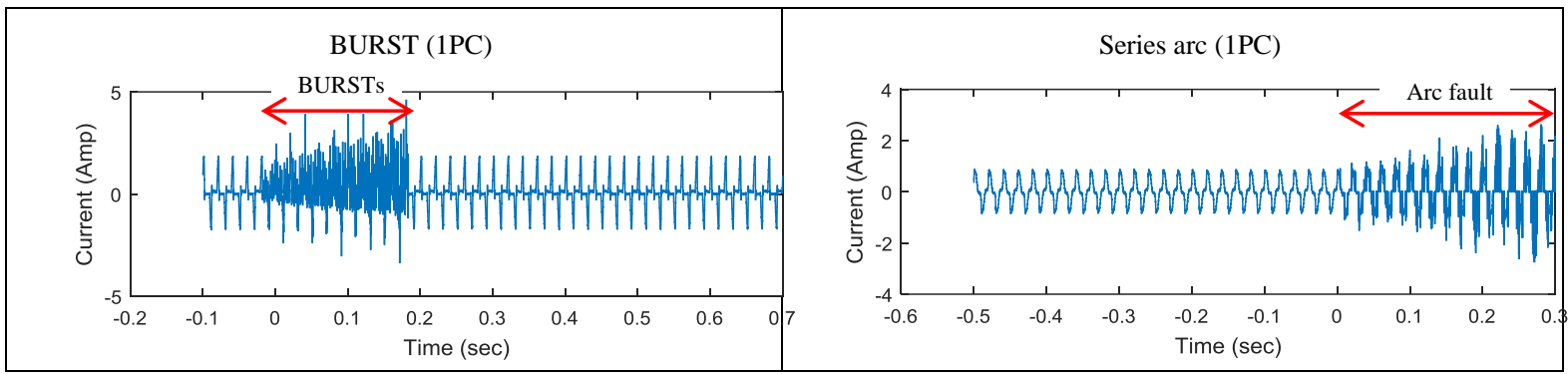

Figure 8. Currents comparison (BURSTs / series arc).
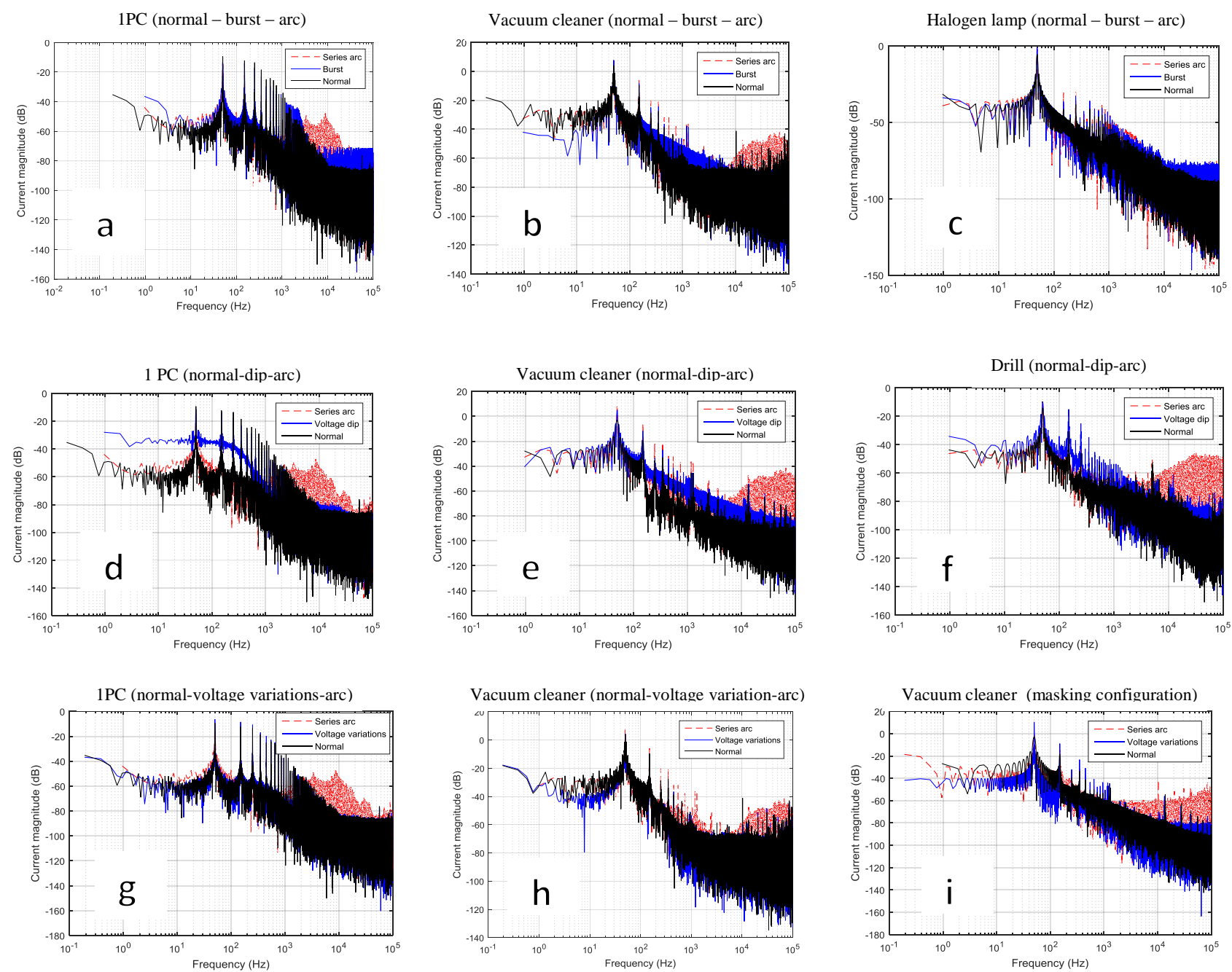

Figure 9. Spectral density for different appliances. 


\section{CONCLUSION}

More and more domestic electronic household appliances produce EMI interferences in electrical networks (switch devices, load variations, motor starting, etc.). These perturbations can be in some cases interpreted as arc faults and causes the tripping of an arc fault circuit breaker (false detections). In this paper experiments have been carried out to generate perturbations according to the UL 1699 and IEC 62606 standards using the EMTEST UCS500 M4 generator. The perturbations generated in this work according to EN 61000-4-4, EN 61000-4-5 and EN 61000-4-11 standards are fast transient perturbations (BURT), surge, voltage dip and voltage variations. The current sensor in a circuit breaker has a limited frequency band. Thus, we limited our analysis to the 0$100 \mathrm{kHz}$ frequency band.

The EMI perturbations have characteristics similar to series arc faults. Frequency analysis shows that the arc fault detection in the presence of EMI perturbations is difficult to achieve if we use a single frequency band for analysis. Thus, the frequency band has to change as a function of different kinds of EMI perturbations and loads.

\section{REFERENCES}

[1] FEMA 2008, Topycal Fire report series: Residential building electrical Fires. United States Fire Administration, 8(2), March 2008.

[2] UL Standard for Safety for Arc-Fault Circuit-Interrupters (UL 1699), April 7, 2006.

[3] International European Standard IEC 62606, Edition 1.0, July, 2013.

[4] X. Zhou, J.J Shea, B. Pahl. "Characterization of EMI/RFI in Comercial and Industrial Electrical Systems". Proceedings of the $59^{\text {th }}$ IEEE Holm Conference on Electrical Contacts. 2013, pp.

[5] International European Standard IEC 61000-4-4-Fast Transient Immunity, Edition 3.0, April, 2012.

[6] International European Standard IEC 61000-4-5-Surge Immunity, Edition 3.0, April, 2012.

[7] International European Standard IEC 61000-4-11-Voltage Dips and Voltage Variations Immunity, Edition 3.0, April, 2012.

[8] CS. Sheng, Y.H Liu, CY. Kuo, YL. Chu, CS. Y, KH. Hsu, CK. Chan, JC. Chang. "Reduction of Conductive EMI Noise Resulted from the Commercial Power Supply". THPPD058 Proceedings of IPAC2012. New Orleans, Lousiana, USA

[9] D.V. Gir, F.M. Tesche, "Classification of Intentional Electromagnetic Environments (IEME)". IEEE Transactions on Electromagnetic Compatibility. 46.3 (2004), pp. 322-328.

[10] S. Gupta, M.S. Reynolds, S.N. Patel, "ElectriSense: Single-Point Sensing Using EMI for Electrical Event Detection and Classification in the Home". UbiComp 2010, September 26-29, 2010, Copenhagen, Denmark.

[11] M. Gulati, S.S. Ram, A. Singh, "An In Depth Study into Using EMI Signatures for Appliance Identification". Proceedings of the First ACM International Conference on Embedded Systems For EnergyEfficient Buildings, 2014, Menphis, TN, USA.

[12] Q. Zhou, Y. Chen, Z. You, "Infrastructure-Mediated Sensing Based Home Appliances Monitoring System using the EMI Characteristics", Chinese Journal of Electronics, 23.3 (2014).

[13] Handbook of EMC Testing and Measurement, IEE Electrical Measurement Series No. 8 By David Morgan (Peter Peregrinus, Stevenage, U.K 1994), 304pp.

[14] Dipak L. Sengupta, Valdis V. Liepa (2006). Applied Electromagnetics and Electromagnetics, New Jersey, Wiley Series in Microwave and Optical Engineering.

[15] N. Hadziefendic, M. Kostic, Z. Radakovic. "Detection of Series Arcing in Low-Voltage Electrical Installations", European Transactions on Electrical Power, 19.3 (2009), pp. 423-432.

[16] P. Muller, S. Tenbohlen, R. Maier, M. Anheuser, "Characteristics of Series and Parallel Low Current Arc Faults in the Time and Frequency Domain", Proceedings of the $56^{\text {th }}$ IEEE Holm Conference on Electrical Contacts, 2010, pp. 1-7.
[17] DW. Park, IK. Kim, SY. Choi, GS. Kil. "Detection Algorithm of Series Arc for Electrical Fire Prediction", International Conference on Monitoring and Diagnosis, 2008, pp. 716-719.

[18] B.M. Aucoin and B.D. Russell. "Distribution High Impedance Fault Detection utilizing High Frequency Current Components", IEEE Power Engineering Review, 1982, pp. 46-47.

[19] D.C. Yu and S.H. Khan. "An Adaptive High and Low Impedance Fault Detection Method", IEEE Transactions on Power Delivery, 9.4 (1994), pp. 1812-1821. 